\title{
On The Integrated Formulation of the Tau Method Involving At Most Two Tau Parameters for Ivps in Odes
}

\author{
A.I.Ma'ali ${ }^{1}$, R.B.Adeniyi ${ }^{2}$ \\ ${ }^{I}$ Department of Mathematics and Computer Science, Ibrahim Badamasi Babangida University, Lapai, Niger \\ State, Nigeria \\ ${ }^{2}$ Department of Mathematics, University of Ilorin, Kwara State. Nigeria
}

Abstract: An error estimation of the integrated variant of the tau method for ordinary differential equations is hereby considered for the class of equations characterized by $m+s \leq 2$ where $m$ and $s$ are, respectively, the order and the number of overdetermination of the differential equation. Some general results are obtained and applied to test problems. Numerical evidences show that the estimate adequately captures the order of the tau approximation.

Key Words: Tau Method, Overdetermination, perturbation, class, Error estimation, Variant

\section{Introduction}

The tau method was originally developed by Lanzcos (1938) for the solution of the m-th order problem

With the conditions

$$
(\mathrm{l}) \equiv \sum_{r}^{m} P_{r}() y^{(r)} \quad \text { ( ) } \quad \leq \leq
$$

$$
L * y\left(x_{r k}\right) \equiv \sum_{r}^{m} a_{r k}(x) y^{(r)}\left(x_{r k}\right) \quad \alpha_{k} \quad k \quad() m
$$

Where $y^{(r)}(x)$ stands for the derivatives of order $y x, f x$ and $P_{r}(x) r=0,1, \ldots, \mathrm{m}$, are polynomials (or polynomial approximations immediately derivable by using the tau method) of given function; where $a_{r k}, x_{r k}$ and $\alpha_{k}$ and given real numbers.

The method solves problem (1) by seeking an approximation:

$$
y_{n}(x) \sum_{r}^{n} a_{r} x^{r} \quad n \quad \infty
$$

This is the exact solution of the perturbed form:

$$
L y_{n}(x) \equiv \sum_{r}^{m} P_{r}(x) y_{n}^{(r)}(x) \quad f(x) \quad H_{n}(x)
$$

With the conditions stated by Eq.(2)

$P_{r}(x) \quad \sum_{k}^{N_{r}} P_{r k} x^{k}$

$H_{n} x$ is a linear combination of Chebyshev polynomials valid in the interval [a, b] and it may be of the form

$$
H_{n}(x) \quad \sum_{r}^{m} T_{m} s-T_{n-m} r \quad x
$$

The parameters in Eq. (6) is the number of over-determinations of Eq.(4). $T_{r}$ 's are the tau parameters to be determined. By inserting (3) into Eq. (4) and then applying the conditions (2), we get the system of linear equation in $(\mathrm{n}+\mathrm{m}+\mathrm{s}+1)$ unknown constants $\mathrm{a}_{\mathrm{r}},(\mathrm{r}=0(1) \mathrm{n}), T \quad T \ldots, T_{m}{ }_{s}$. This system is then solved to obtain the $(n+m+s+1)$ unknown constants which are to be substituted into Eq. (2) in order to get our approximate solution of Eq. (1).

Lanzcos introduced the use of the canonical polynomials $Q_{r}(\mathrm{x}), \mathrm{r} \geq 0$,

$L Q_{r}(\mathrm{x})=x^{r}$

Where $\mathrm{L}$ is the linear operator

\section{$L \quad \sum_{r}^{m} \quad P_{r}(x) \frac{d^{r}}{d x^{r}}$}

The expression of the approximate solution $y_{n} x$ in terms of a canonical polynomials offers several advantages because it does not depend on the boundary condition of the problems which we want to solve nor 
on the interval in which the solution is sought, allowing for every segmentation of the domain. They are permanent in the sense that if an approximation of higher degree is required, the computation does not need to be repeated from the beginning. Furthermore, the tau method does not require a stage of discretisation of the given differential operator; as discrete variable method do.

A recursive generation of polynomial was introduced by Ortiz (1969) to give some flexibility in the computation of the conical polynomials.

An approach developed for an improved accuracy of the approximation $y_{n} x$ of $y x$, is the integrated function, whereby we first integrate through Eq. (1), to have

$$
\begin{aligned}
& I_{L}(()) \quad \iint m \int \sum_{r}^{m} P_{r}(x) y^{(r)}(x) d x d x \ldots d x \\
& \quad \iint m \int \sum_{r}^{m} f_{r} x^{r} \sum_{r}^{s^{s-}} T_{m} s_{-} T_{n-m} \quad r \quad x \quad d x d x \ldots d x
\end{aligned}
$$

Thus,

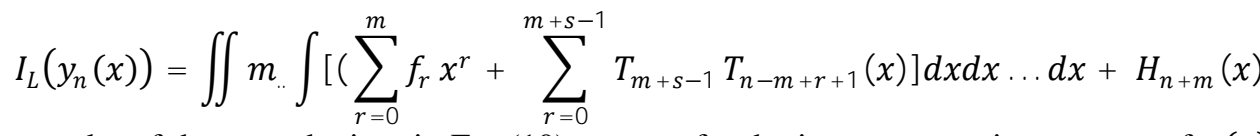

the higher order of the perturbation in Eq. (10) account for the improvement in accuracy of $y(x)$ in contrast of the differential and recursive formulations.

\subsection{DEFINATION OF TERMS}

Definition 1.1.1

A differential equation (or a system of differential equation) together with its associated given conditions will be referred to a Differential system.

\section{Definition 1.1.2}

The differential system

$$
\begin{array}{rllll}
L y_{n}(x) & \equiv \sum_{r}^{m} P_{r}(x) y^{(r)}(x) & f(x) & H_{n}(x) & \\
L * y_{n}\left(x_{r k}\right): & \sum_{r}^{m} a_{r k} y_{n}^{(r)}\left(x_{r k}\right) & \alpha_{r k} & k & (\text { ( ) } m
\end{array}
$$

will be called the Tau problem corresponding to the differential system

$$
\begin{aligned}
& \operatorname{Ly}(x) \quad \sum_{r}^{m} P_{r}(x) y^{(r)}(x) \quad f(x) \\
& L * y\left(x_{r k}\right): \quad \sum_{r}^{m-} a_{r k} y^{(r)}\left(x_{r k}\right) \quad \propto_{k} \quad k \quad(\text { ) } m
\end{aligned}
$$

We call the $\mathrm{n}$-th degree polynomial, $y_{n} x$, which satisfies the Tau problem (12), the tau approximant of (13) and the tau solution of Eq. (12) resulting in the process of solution of (13) will be referred to as tau system of problem (12).

\section{Definition 1.1.3}

The system of equation $\mathrm{A} \underline{\tau}=\mathrm{B}$ where $\underline{\tau}=\left(a, a, a, \ldots \ldots, a_{n}, \tau, \tau, \tau, \ldots \tau_{m}{ }_{s}\right)^{\mathrm{T}}$,

Resulting the process of solution tau of (13) will be referred to as Tau System of (12)

\section{Definition 1.1.4}

The number of over-determination of the DE (1) is defined by $\mathrm{S}=\max \left\{\mathrm{N}_{\mathrm{r}}-\mathrm{r}: 0 \leq \mathrm{r} \leq \mathrm{m}\right\} \geq$ for $N_{r} \geq r$

\section{Derivation Of Tau Approximant} problem:

We consider here the derivation of tau approximants of varying orders and degrees, for the class of

$$
\begin{aligned}
& L y(x) \quad \sum_{r}^{m} P_{r}(x) y^{(r)}(x) \quad f(x) \quad a \leq x \leq b \\
& L * y\left(x_{r k}\right): \quad \sum_{r}^{m-} a_{r k} y^{(r)}\left(x_{r k}\right) \quad \propto_{k} \quad k \quad()(m-)
\end{aligned}
$$


where,

$$
P_{r}(x) \quad \sum_{k}^{N_{r}} P_{r k} x^{k}
$$

The amount of work as well as the size of the space this will involve is enormous; we shall illustrate the procedure for a fifth degree approximant and then provide only the results for approximants of other degrees. In the work, we shall derive a fifth degree approximant for

\section{The case $m=1, s=0$}

$$
L y_{n}(x) \equiv \sum_{r}^{m} P_{r}(x) y_{n}^{(r)}(x) \quad \sum_{r}^{n} f_{r} x^{r} \quad H_{m}(x)
$$

From (1) above, the general case for $\mathrm{m}=1$ and $\mathrm{s}=0$ is given by;

$\operatorname{Ly}(\mathrm{x}) \equiv\left(\begin{array}{llllllll}P & P & x\end{array}\right) y^{\prime}(x) \quad P \quad y(x) \quad \sum_{i}^{n} f_{r} x^{r} \quad \tau T_{n}(x)$

And from (11) we have

$$
I_{L} y(x) \quad \int^{x}\left(\begin{array}{lll}
P & P & t
\end{array}\right) d t \quad \int^{x} p \quad y t d t \quad \int^{x} \sum_{r}^{n} f_{r} t^{r} d t \quad \tau T_{n}(x)
$$

where,

$T_{n}(x)=\sum_{r}^{n} C_{r}^{n} x^{r}$

This leads to

$$
P \quad\left[y_{n}(x)-\propto\right] \quad P \quad x y_{n} x-\int^{x} y_{n}(t) d t \quad P \quad \int^{x} y_{n}(t) d t \quad \int^{x} \sum_{r}^{n} f_{r} t^{r} d t \quad \tau \sum_{r}^{n} C_{r}^{n} x^{r}
$$

We seek an approximant solution of the form $y_{n}(x) \quad \sum_{r}^{n} a_{r} x^{r}$

With (23), (22) now becomes,

$$
\begin{aligned}
P \sum_{r}^{n} a_{r} x^{r}-P & \propto P \sum_{r}^{n} a_{r} x^{r} \\
& -\sum_{r}^{n} a_{r} \frac{x^{r}}{r} \quad P \sum_{r}^{n} a_{r} \frac{x^{r}}{r} \quad \sum_{r}^{n} f_{r} \frac{x^{r}}{r} \quad \tau \quad a \sum_{r}^{n} C_{r}{ }^{n} X^{r}
\end{aligned}
$$

This gives

$$
P \sum_{r}^{n} a_{r} x^{r} \sum_{r}^{n}\left[\frac{P}{r}\right] a_{r} x^{r}-\tau \sum_{r}^{n} C_{r}{ }^{n} x^{r} \sum_{r}^{n} f_{r} \frac{x^{r}}{r} \quad P \propto
$$

Thus, for example, when $\mathrm{n}=5$, we have:

$$
P \sum_{r} a_{r} x^{r} \sum_{r} \frac{P{ }^{r} P}{r} a_{r} x^{r}-\tau \sum_{r} C_{r}{ }^{n} x^{r} \sum_{r}^{n} f_{r} \frac{x^{r}}{r} \quad P \propto
$$

Equating corresponding coefficients power of $\mathrm{x}$, we obtain the tau system

$$
\left(\begin{array}{ccccccc}
P_{10} & 0 & 0 & 0 & 0 & 0 & -C_{0}^{(5)} \\
P_{00} & P_{10} & 0 & 0 & 0 & 0 & -C_{1}^{(5)} \\
0 & N_{32} & 0 & 0 & 0 & 0 & -C_{2}^{(5)} \\
0 & 0 & N_{43} & P_{10} & 0 & 0 & -C_{3}^{(5)} \\
0 & 0 & 0 & N_{54} & P_{10} & 0 & -C_{4}^{(5)} \\
0 & 0 & 0 & 0 & N_{65} & P_{10} & -C_{4}^{(5)} \\
0 & 0 & 0 & 0 & 0 & N_{76} & 0
\end{array}\right)\left(\begin{array}{c}
a_{0} \\
a_{1} \\
a_{2} \\
a_{3} \\
a_{4} \\
a_{5} \\
\tau_{1}
\end{array}\right)=\left(\begin{array}{c}
P_{10} \alpha_{0} \\
f_{0} \\
f_{1} / 2 \\
f_{2} / 3 \\
f_{3 / 4} \\
f_{4 / 5} \\
f_{5 / 6}
\end{array}\right) \quad(27)
$$

Continuing with the process, using $\mathrm{m}=1$ and $\mathrm{s}=1$. By expanding (27), we obtain the following tau system For $\mathrm{m}=1, \mathrm{~s}=1$, we have,

$$
\left(\begin{array}{cccccccc}
P_{10} & 0 & 0 & 0 & 0 & 0 & -C_{0}^{(7)} & -C_{0}^{(6)} \\
P_{00} & P_{10} & 0 & 0 & 0 & 0 & -C_{1}^{(7)} & -C_{1}^{(6)} \\
\frac{P_{01}}{2} & N_{32} & P_{10} & 0 & 0 & 0 & -C_{2}^{(7)} & -C_{1}^{(6)} \\
0 & N_{42} & N_{43} & P_{10} & 0 & 0 & -C_{3}^{(7)} & -C_{3}^{(6)} \\
0 & 0 & N_{53} & N_{54} & P_{10} & 0 & -C_{4}^{(7)} & -C_{4}^{(7)} \\
0 & 0 & 0 & N_{64} & N_{65} & P_{10} & -C_{4}^{(7)} & -C_{4}^{(7)} \\
0 & 0 & 0 & 0 & N_{75} & N_{76} & -C_{4}^{(7)} & -C_{4}^{(7)} \\
0 & 0 & 0 & 0 & 0 & N_{65} & -C_{4}^{(7)} & 0
\end{array}\right)\left(\begin{array}{c}
a_{0} \\
a_{1} \\
a_{2} \\
a_{3} \\
a_{4} \\
a_{5} \\
\tau_{1} \\
\tau_{1}
\end{array}\right)=\left(\begin{array}{c}
P_{10} \alpha_{0} \\
f_{0} \\
f_{1} / 2 \\
f_{2 / 3} \\
f_{3} / 4 \\
f_{4} / 5 \\
f 5 / 6 \\
0
\end{array}\right)
$$


where

$\mathrm{N}_{32}=\frac{P}{P}, \mathrm{~N}_{42}=\frac{P}{P}, \mathrm{~N}_{43}=\frac{P \quad P}{P}, \mathrm{~N}_{53}=\frac{P}{P}, \mathrm{~N}_{54}=\frac{P \quad P}{\longrightarrow}, \quad \mathrm{N}_{64}=\frac{P \quad P}{P}, \quad \mathrm{~N}_{65}=\frac{P P}{P}$,

$\mathrm{N}_{75}=\frac{P \quad P}{P}, \mathrm{~N}_{76}=\frac{P \quad P}{P}, \mathrm{~N}_{86}=\frac{P \quad P}{}$

The case $m=2, s=0$

From (1) the general case for $\mathrm{m}=2$ and $\mathrm{s}=0$ is given by

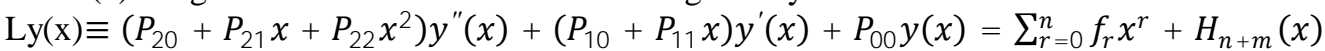

$y(\mathrm{I})=\alpha, \mathrm{y}^{\prime}(0)=\alpha, \alpha \leq \mathrm{x} \leq \mathrm{b}$

where,

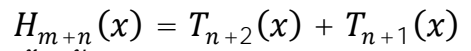

$$
\begin{aligned}
& \int^{x} \int^{u}\left(\begin{array}{lllll}
P & P & t & P & t
\end{array}\right) y_{n}^{\prime \prime}(t) d t d u \quad \int_{0}^{x} \int^{u} P \quad P \quad P \quad y_{n}{ }^{\prime}(t) d t d u \\
& \int^{x} \int^{u}\left(\begin{array}{lll}
P & y_{n} & t
\end{array}\right) d t d u \quad \int^{x} \int^{u} \sum_{r}^{n} f_{r} x^{r} \quad \tau T_{n} \quad x \quad \tau T_{n} \quad x
\end{aligned}
$$

We integrate the terms in (31) to have,

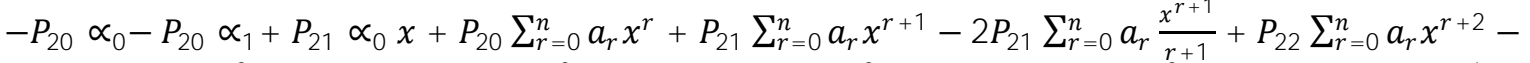

$$
\begin{aligned}
& P \quad \sum_{r}^{n} a_{r} \frac{x^{r}}{r} \quad P \quad \sum_{r}^{n} a_{r} \frac{x^{r}}{r} \quad P \quad \sum_{r}^{n} a_{r} \frac{x^{r}}{r}-P \quad \sum_{r}^{n} a_{r} \frac{x^{r}}{r} \quad P \quad \sum_{r}^{n} a_{r} \frac{x^{r}}{r} \\
& P \quad \sum_{r}^{n} \quad a_{r} \frac{x^{r}}{r \quad r}-\tau \sum_{r}^{n}-C_{r}^{n} x^{r}-\tau \sum_{r}^{n}-C_{r}^{n} x^{r} \\
& \sum_{r}^{n} f_{r} \frac{x^{r}}{r \quad r}
\end{aligned}
$$

This gives,

$\sum_{r}^{n} \quad P \quad a_{r} x^{r} \quad \sum_{r}^{n}\left[\frac{(r-) P}{r} \quad P \quad\right] a_{r} x^{r} \quad \sum_{r}^{n}\left[\begin{array}{lll}P & r P & (r-r) P \\ r & r\end{array}\right] a_{r} x^{r} \quad-$

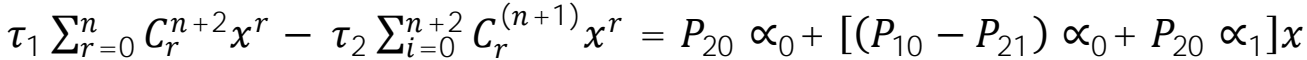

$\sum_{r}^{n} f_{r} \frac{x^{r}}{r \quad r}$

Equating the corresponding coefficients of powers of $\mathrm{x}$ in (33) when $\mathrm{n}=5$ we have the tau system

$\mathrm{D}=$

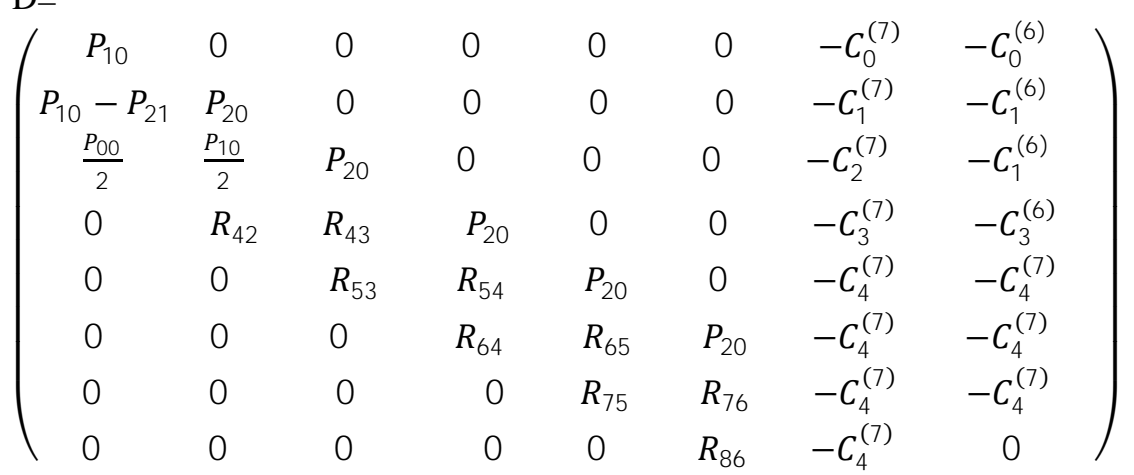

$\mathrm{E}=\left(\begin{array}{l}a \\ a \\ a \\ a \\ a \\ a \\ \tau \\ \tau\end{array}\right) \quad \mathrm{F}=\left(\begin{array}{c}P \propto \\ f \\ f / \\ f / \\ f / \\ f / \\ f /\end{array}\right)$

Then, for $m=2, s=0$ and $n=5$

$\mathrm{DE}=\mathrm{F}$

where, 


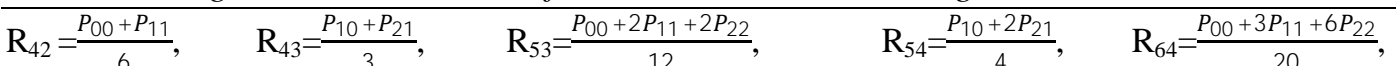

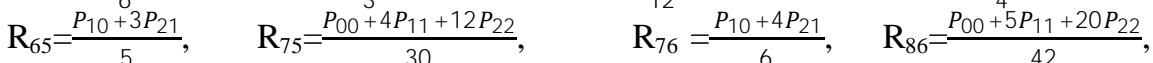

$$
\begin{aligned}
& \text { and, } \\
& \mathrm{a}_{\mathrm{kk}}=\mathrm{P}_{\mathrm{m} 0,}, \forall \mathrm{k}=1(1)(\mathrm{n}+1) \quad \forall \mathrm{m} \text {. }
\end{aligned}
$$

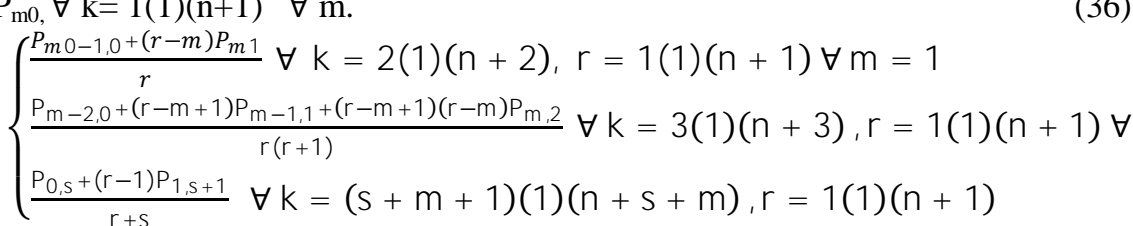

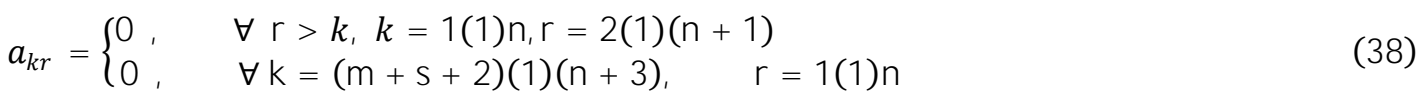

$$
\begin{aligned}
& \begin{array}{lll}
a_{k n} & -C_{k-}^{n} & \mathrm{k}=1(1)(\mathrm{n}+3) \\
a_{k n} & -C_{k-}^{n} & \mathrm{k}=1(1)(\mathrm{n}+2)
\end{array} \\
& b \quad P_{m} \propto \quad \forall m \\
& b \overline{(m-)}\left\{\propto \sum_{r}-{ }^{r} r P_{r} \quad r \quad \propto \sum_{r}-{ }^{r} r P_{r} \quad r \quad \forall m\right\} \\
& b_{i}\left\{\begin{array}{llll}
\frac{f_{i-}}{i-} & \forall m & \forall i & \text { ( ) } n \\
\frac{f_{i-}}{i-i-} \forall m & \forall i & \text { ( ) } n
\end{array}\right.
\end{aligned}
$$

III. Procedure For Error Estimation

The integrated formulation of the tau method often leads to better accuracy of the tau solution. (See Fox(1962) and Ortiz(1993)). To this end, let $\iint . .^{i} \int g(x) d x$ denote the indefinite integration i times applied to the function $\mathrm{g}(\mathrm{x})$ and let

The integration form of

$$
I_{L} \quad \iint \ldots{ }^{m} \int L() d x
$$

$\left.\mathrm{L} e_{n}(\mathrm{x})\right)=-\mathrm{H}_{\mathrm{n}}(\mathrm{x}) \mathrm{dx}$

is therefore

$$
I_{L}\left(e_{n}(x)\right) \quad-\iint \ldots{ }^{m} \int H_{n} x d x
$$

We considered the perturbed form of (42) i.e. the perturbed integrated error equation

which is equivalent to

$$
I_{L}\left(e_{n}(x)\right)_{n} \quad-\iint \ldots{ }^{m} \int H_{n} x d x \quad \hat{H}_{m} n \quad x
$$

and which is satisfied by $\left(e_{n}(x)\right)_{n}$, given by

$$
\begin{gathered}
I_{L}\left(e_{n}(x)\right)_{n} \quad-\iiint_{n^{m}} \int^{m} \sum_{r}^{m} T_{m}{ }^{s-}-T_{n} r \quad x \quad C_{m} x d x \\
\sum_{r}^{m-} T_{m} T_{s-} T_{n} r \quad(x)
\end{gathered}
$$

$$
\left(e_{n}(x)\right)_{n} \quad \frac{\mu_{m}(x) \emptyset_{n} T_{n-m} \quad x}{C_{n-m}^{n-m}}
$$

With $\emptyset_{n}$ replaced by $\widehat{\emptyset}_{n}$ where ,

$$
\begin{aligned}
& \hat{H}_{m} n \\
& \text { (x) } \quad \tilde{\tau} T_{n}^{*} \mathrm{~ms}
\end{aligned}
$$

We insert (48) in (47) and then equate the coefficient of $x^{n+m+s+1}, x^{n+m+s}, \ldots, x^{n-m}$ 

for the determination of the parameter $\widehat{\emptyset}_{n}$ of $\left(e_{n}(x)\right)_{n}$. We then have

as an estimation of $\varepsilon$

$$
\varepsilon^{*} \frac{\left|\widehat{\emptyset}_{n}\right|}{n-m}
$$

We shall carry out these steps for obtaining $\emptyset_{n}$ with various values of $\mathrm{m}$ and $\mathrm{s}$ and then generalize the result to obtain a recursive formular for $\emptyset_{n}$.

\section{Error Estimation For The Integrated Formulation}

\section{The case $m=1, s=0$}

From (46) we have for the problem:

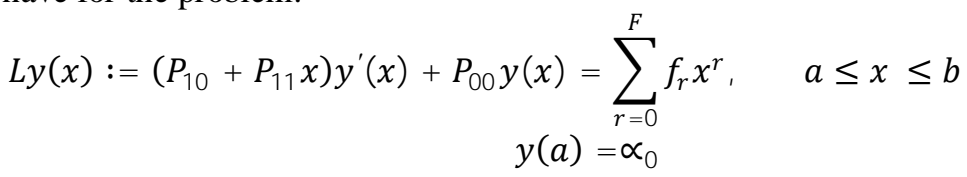

The equation :

$$
\begin{aligned}
L y(x):=\int^{x}\left(\begin{array}{lllll}
P & P & u
\end{array} e_{n}^{\prime}(u)_{n} d u \int^{x} P e_{n}^{\prime}(u)_{n} d u\right. \\
-\tau \int^{x} \sum_{r}^{n} C_{r}{ }^{n} u^{r} d u \quad \tau \sum_{r}^{n} C_{r}{ }^{n} x^{r}
\end{aligned}
$$

where

that is,

$$
\left(e_{n}(x)\right)_{n} \quad \frac{x T_{n} x \emptyset_{n}}{C_{n}^{n}}
$$

$$
\left(e_{n}(x)\right)_{n} \quad \frac{\emptyset_{n}}{k} K x^{n} \quad K x^{n} \quad K x^{n-}
$$

Where $k \quad C_{n}{ }^{n}, k \quad C_{n-}^{n}, k \quad C_{n-}^{n}$

Now,

$$
\int^{x}\left(e_{n}(x)\right)_{n} \quad \frac{\emptyset_{n}}{k}\left[\frac{k x^{n}}{n} \quad \frac{k x^{n}}{n} \quad \frac{k x^{n}}{n}\right]
$$

Inserting (54) and (55) into (52) gives

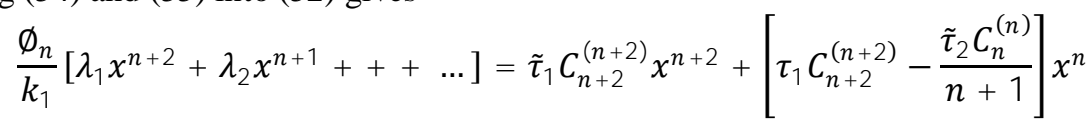

where,

$$
\lambda \quad\left[\begin{array}{lll}
P & (n & n
\end{array}\right] k \quad \lambda \quad P \quad k\left[\begin{array}{lll}
P & (n & ) P \\
n
\end{array}\right] k
$$

Equating coefficient of corresponding powers of $x$ from both sides of (52), gives

$$
\tilde{\tau} C_{n}^{n} \quad \frac{\emptyset_{n} \lambda}{k}
$$

$$
\tilde{\tau} C_{n}^{n} \quad-\frac{\tau k}{n} \quad \frac{\emptyset_{n} \lambda}{k}
$$

From (57) we have

$$
\tilde{\tau} \frac{\emptyset_{n} \lambda}{k C_{n}^{n}}
$$

Inserting this into (59) gives

$\emptyset_{n} \frac{\tau k}{n \quad R}$

where,

$$
R \quad \lambda-\frac{\lambda C_{n}{ }^{n}}{C_{n}{ }^{n}}
$$

Let $R_{1}=\lambda$, then $R_{2}$ can be put in the following recursive form:

$\mathrm{R}_{1}=\lambda$ 


$$
R \quad \lambda-\frac{C_{n}{ }^{n} R}{C_{n}{ }^{n}}
$$

\section{The case $m=1 \mathrm{~s}=1$}

From (46), the most general form for $\mathrm{m}=1$ and $\mathrm{s}=1$ is given by

where $\left(e_{n}(x)\right)_{n} \quad$ in (65) is defined by (48).

$$
\begin{aligned}
\int^{x}\left(\begin{array}{lllll}
P & P & u & P & u
\end{array}\right) e_{n}^{\prime}(u)_{n} d u \int^{x} P \quad P \quad u e_{n}^{\prime}(u)_{n} d u \\
-\tau \int^{x} \sum_{r}^{n} C_{r}{ }^{n} u^{r} d u-\tau \int^{x} \sum_{i}^{n} C_{r}{ }^{n} u^{r} d u \quad \tau \sum_{r}^{n} C_{r}{ }^{n} x^{r} \\
\tau \sum_{r}^{n} C_{r}{ }^{n} x^{r}
\end{aligned}
$$

Thus, Inserting $\left(e_{n}(x)\right)_{n} \quad$ and its integral into (65) gives

where,

$$
\begin{aligned}
& \frac{\emptyset_{n}}{k}\left[\begin{array}{llll}
\lambda x^{n} & \lambda x^{n} & \lambda x^{n} & \ldots
\end{array}\right] \\
& \tilde{\tau} C_{n}{ }^{n} \quad x^{n} \quad\left[\begin{array}{llll}
\left.\tilde{\tau} C_{n}^{(n} \quad\right) & \left.\tilde{\tau} C_{n}^{(n} \quad\right) & -\frac{\left.\tau C_{n}^{(n}\right)}{n}
\end{array}\right] x^{n}
\end{aligned}
$$

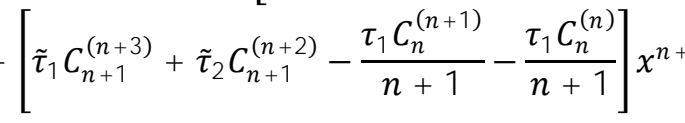

$$
\begin{aligned}
& \lambda \frac{P \quad n \quad P}{n} k \\
& \lambda\left[\frac{P \quad(n+) P}{n}\right]^{n} k \frac{P}{n} k \\
& \lambda \quad P \quad k \quad\left[\begin{array}{lll}
P \quad(n & ) P \\
n
\end{array}\right] k \quad \frac{P \quad n \quad P}{n} k
\end{aligned}
$$

$k \quad C_{n}{ }^{n}, k \quad C_{n-}^{n}, k \quad C_{n-}^{n}$

Equating coefficients of corresponding powers of from both sides of (66), we obtain the following system of equations

$$
\begin{aligned}
& \tilde{\tau} C_{n}{ }^{n} \quad \frac{\emptyset_{n} \lambda}{k} \\
& \tilde{\tau} C_{n}{ }^{n} \quad \tilde{\tau} C_{n}{ }^{n} \quad-\frac{\left.\tau C_{n}^{(n)}\right)}{n} \quad \frac{\emptyset_{n} \lambda}{k} \\
& \tilde{\tau} C_{n}{ }^{n} \quad \tilde{\tau} C_{n}{ }^{n} \quad-\frac{\left.\tau C_{n}^{(n}\right)}{n}-\frac{\tilde{\tau} C_{n}^{(n)}}{n} \frac{\emptyset_{n} \lambda}{k}
\end{aligned}
$$

From (68) we have

$$
\tilde{\tau} \frac{\emptyset_{n} \lambda}{k C_{n}^{n}}
$$

Inserting (71) into (69) and solving for $\tau$ we have

$$
\tau \frac{\tau C_{n}^{n}}{n C_{n}^{n}} \frac{\emptyset_{n}}{k} \lambda-\frac{\tau C_{n}^{(n)} \lambda}{\left.C_{n}^{(n} \quad\right)}
$$

Inserting () and () into () and solving for we obtain

$\emptyset_{n} \quad \frac{k \tau}{n \quad R}$

where

$$
R \quad \lambda-\frac{C_{n}{ }^{n} \quad R}{C_{n}{ }^{n}}-\frac{C_{n}{ }^{n} \quad R}{C_{n}{ }^{n}}
$$

Thus, we have the following recursive form,

$$
R \quad \lambda
$$




$$
R \quad \lambda-\frac{C_{n}^{n} \quad R}{C_{n}{ }^{n}}
$$

We repeat he analysis discussed above with $\mathrm{m}=2$ and thus, we obtain the following results:

\section{For $\mathbf{m}=\mathbf{2}, \mathbf{s}=\mathbf{0}$}

$\emptyset_{n} \frac{-k \tau_{m} s}{n n \quad R}$

Thus, we obtain general expression for $\emptyset_{n}$ as:

$\emptyset_{n} \frac{k \tau}{\prod_{r}^{m} n s r R_{m} s} \quad \forall m \quad s$

Where $k \quad C_{n m-}^{n}{ }^{m-s}$

$$
\emptyset_{n} \quad \frac{k \tau}{\prod_{r}^{m} n s \quad r-R_{m} s} \quad \forall m \quad s
$$

Where $R_{m+s+1}$ is given recursively in terms of $R_{1}, R_{2}, R_{3}, \ldots, R_{m+s}$ as follows:

$\mathrm{R}_{1}=\lambda$

$$
R_{u} \quad \lambda_{u}-\sum_{i}^{n} \frac{C_{n u--s}^{n u-i}}{C_{n u-}^{n u-}} R_{i} \quad u \quad \ldots m \quad s
$$

and

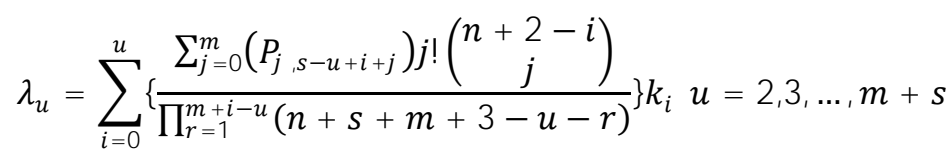

Provided $\mathrm{i} \geq \mathrm{u}-\mathrm{m}+1$

Thus, from (50), we have the following expression for $\varepsilon^{*}$ :

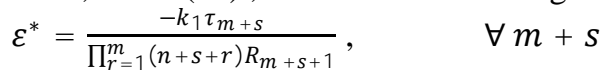

where $k=C_{n-m}^{n-m} \quad$ and

$\varepsilon^{*} \quad \frac{-k \tau_{m} s}{\prod_{r}^{m} n s \quad r-R_{m} s}, \quad \forall m \quad s$

\section{Numerical Examples}

We consider here some selected examples for experimentation with our results of the preceeding section for $\mathrm{m}+\mathrm{s}=1$ and $\mathrm{m}+\mathrm{s}=2$, the exact error is defined as

where $\left\{x_{k}\right\}=\{0.01 \mathrm{k}\}$, for $\mathrm{k}=0(1) \leq 100$

$$
\xi_{\ell} \max _{a \leq x \leq b}\left\{\left|y x_{k}-y_{n} x_{k}\right|\right\}
$$

The numerical results are presented in the tables bellow the examples

Problem 4.1
$y^{\prime \prime}(x)-y \quad x=0$
$y=1, \quad y^{\prime} x=1$,
$y(x) \quad e^{x}$
$0 \leq x \leq 1$

Table 4.1

Error and error estimation for problem 4.1

\begin{tabular}{|l|l|l|l|l|}
\hline Error $\mathrm{n}$ & 2 & 3 & 4 & 5 \\
\hline Estimates & $4.61 \times 10^{-3}$ & $7.50 \times 10^{-5}$ & $9.46 \times 10^{-7}$ & $5.34 \times 10^{-8}$ \\
\hline Exact & $3.67 \times 10^{-3}$ & $1.01 \times 10^{-5}$ & $4.53 \times 10^{-7}$ & $2.37 \times 10^{-8}$ \\
\hline
\end{tabular}

\section{Problem 4.2}

$y^{\prime} x-x y x=1-x$

$y()=0$

$0 \leq x \leq 1$

Table 4.2

Error and error estimation for example 3.2

\begin{tabular}{|l|l|l|l|l|}
\hline Error $\mathrm{n}$ & 2 & 3 & 4 & 5 \\
\hline Estimates & $6.01 \times 10^{-3}$ & $6.7 \times 10^{-4}$ & $3.24 \times 10^{-4}$ & $4.6 \times 10^{-5}$ \\
\hline Exact & $9.34 \times 10^{-2}$ & $7.11 \times 10^{-4}$ & $4.89 \times 10^{-4}$ & $4.35 \times 10^{-5}$ \\
\hline
\end{tabular}


Problem 4.3

\begin{tabular}{lrll}
\hline$y^{\prime}(x)$ & $x y(x)$ & & $y(\mathrm{x})=\mathrm{e}^{\mathrm{x}}$ \\
$y()$ & $y(x)$ & $e^{-x}$ & $0 \leq \mathrm{x} \leq 1$
\end{tabular}

Table 4.3

Error and error estimation for problem 3.3

\begin{tabular}{|l|l|l|l|l|}
\hline Error $\mathrm{n}$ & 2 & 3 & 4 & 5 \\
\hline Estimates & $3.51 \times 10^{-3}$ & $4.60 \times 10^{-4}$ & $5.65 \times 10^{-5}$ & $9.02 \times 10^{-6}$ \\
\hline Exact & $1.34 \times 10^{-2}$ & $2.37 \times 10^{-4}$ & $3.1 \times 10^{-5}$ & $4.67 \times 10^{-6}$ \\
\hline
\end{tabular}

\section{Problem 4.4}

$y^{\prime \prime}(x) \quad y(x) \quad x$
$y \quad=0, y^{\prime}(0)=3 \quad y x=\begin{gathered}0 \leq x \leq 1 \\ x\end{gathered} \quad x \quad x-$

\section{Table 4.4}

Error and error estimation for problem 4.4

\begin{tabular}{|l|l|l|l|l|}
\hline Error $\mathrm{n}$ & 2 & 3 & 4 & 5 \\
\hline Estimates & $6.46 \times 10^{-4}$ & $4.23 \times 10^{-5}$ & $7.0 \times 10^{-7}$ & $3.85 \times 10^{-8}$ \\
\hline Exact & $8.84 \times 10^{-3}$ & $1.06 \times 10^{-5}$ & $2.86 \times 10^{-6}$ & $2.14 \times 10^{-7}$ \\
\hline
\end{tabular}

\section{Conclusion}

The integrated formulation of the tau method and its error estimation have been generalized for those ODEs, whose perturbed form involves a maximum of two tau parameters and consequently a maximum overdetermination number, $\mathrm{s}$, and to one.

The tau system for the determination of the tau approximation $y_{n} x$ was first constructed. The error estimation which followed immediately provides the estimate of the error in $y_{n} x$. Numerical evidences, obtained for some selected problems, revealed that the estimate accurately captures the order of the exact error.

\section{References}

[1]. Adeniyi R.B. (2008): An improved error estimation of the tau method for Boundary value problems. Research journal of applied sciences. Vol.3, issue 6 pp 456-464.

[2]. Adeniyi R.B. (2000): optimality of error estimate of one-step Numerical International journal of computer Mathematics, Vol.75 issue 3 pp 283-295.

[3]. Adeniyi R.B, Onumanyi P. (1991): error estimation in the numerical solution of the ordinary differential equation with the tau method. Computer and mathematics with application Vol.21, pp 19 - 21 (Available online 4 sept 2002).

[4]. Adeniyi R.B. (1991): On the tau method for numerical solution of ordinary differential equation doctoral Thesis, University Ilorin.

[5]. Adeniyi R.B, Onumanyi P and Taiwo O.A (1990): A computational error estimation of the tau method for non-linear differential equation. Journal of Nigeria mathematics Soc. Vol.9, pp $21-32$.

[6]. Adeniyi R.B, (1985): An error estimation technique for linear ordinary differential equations using the tau method. M. Sc dissertation (unpublished), University of Ilorin.

[7]. Banks H.T and Wade J.G (1991): Weak tau approximations for distributed parameter system in inverse problems. Numer. Funct. Anal. Optima, Vol.12 pp 1 -31.

[8]. Egbetade S.A (200): A computational error estimate of he tau method. Ife journal of Science, Vaol.82 pp $105-110$.

[9]. Fox, L. (1962): Chebyshev Methods for Ordinary Differential Equations. Comp. J. 4: 318 - 331

[10]. Hussaini S.M (2009): The Adaptive operational tau method for system of ordinary differential equations. Journal of computernal and applied mathematics, Vol.3 issue 1, Sept 2009.

[11]. Namasivayam S. and Ortiz E.L (1993): Error analysis of the tau method. Dependence of the approximation error on the choice of perturbation term. Computer and mathematics with application, Vol.25, pp $89-104$

[12]. Onumanyi P and Ortiz E.L (1984): Numerical solution of stiff and singularly perturbed BVP with a segmented adaptive formulation of the tau method. Mathematics of computation Vol.343 pp $189-203$.

[13]. Onumanyi P and Ortiz E.L (1984): Numerical solutions of higher order boundary value problem for ordinary differential equations with an estimates of the error. Inter. J. Numer. Mech. Engrg. Vol. 18 pp $775-781$

[14]. Ortiz E.L (1969): the tau method. SIAM. J. Numer. Anal. Vol.6, pp $480-492$. 